\title{
MicroRNAs present in neuron-derived extracellular vesicles extracted from the blood of psychiatric patients as potential biomarkers of mood disorders
}

\author{
Marta Dziedzicka-Wasylewska, Joanna Solich, Agata Faron-Górecka \\ Department of Pharmacology, Maj Institute of Pharmacology, Polish Academy of Sciences, Kraków, Poland \\ Correspondence to: Marta Dziedzicka-Wasylewska. Department of Pharmacology, Maj Institute of Pharmacology, Polish Academy of Sciences, Kraków, \\ Poland. Email: marta.dziedzicka-wasylewska@uj.edu.pl. \\ Comment on: Saeedi S, Nagy C, Ibrahim P, et al. Neuron-derived extracellular vesicles enriched from plasma show altered size and miRNA cargo as a \\ function of antidepressant drug response. Mol Psychiatry 2021. [Epub ahead of print]. doi:10.1038/s41380-021-01255-2.
}

Received: 15 October 2021; Accepted: 16 November 2021; Published: 30 December 2021.

doi: $10.21037 /$ exrna-21-22

View this article at: https://dx.doi.org/10.21037/exrna-21-22

Mood disorders, including major depressive disorder (MDD), are still a considerable burden for modern society. Despite the availability of approx. fifty different drugs, the treatment of MDD is far from ideal, nor is the full understanding of the mechanism of action of antidepressant drugs (ADs) administered repeatedly (which is necessary to achieve clinical efficacy). There have been several hypotheses explaining the etiopathogenesis of depression. Most of these are based on the mechanisms of action of known ADs, the first of which were discovered by serendipity in the 1950s (imipramine and iproniazid). Later, the mechanism of their acute action (e.g. non-selective inhibition of re-uptake of serotonin and noradrenaline, and inhibition of monoamine oxidase, respectively) has been elucidated, and most of the newly synthesized compounds mimic some or all of their features: being either more selective towards one of the biogenic amines, having an affinity towards various neurotransmitter receptors such as $\mathrm{N}$-methyl D-aspartate (NMDA) receptors for glutamate, or being more selective and reversible monoamine oxidase inhibitors (1). Knowing these mechanisms does not help in achieving a satisfactory clinical outcome, in fact, we still do not understand the etiopathology of MDD and other mood disorders. Most of the numerous behavioral, biochemical, and molecular preclinical studies focus on various kinds of stress since it is regarded as the main factor contributing to mood disorders. Stress is defined as a condition that seriously perturbs the psychological and physiological balance of an individual. Traumatic stress, however, does not affect everyone in the same manner-some susceptible individuals (not only humans but also animals) adapt poorly to stressors and express inappropriate responses that can become persistent states of stress themselves. Resilient individuals can perceive adversity and develop adaptive psychological and physiological responses. The underlying mechanisms of these responses are not fully understood, although they are known to depend on a combination of genetic and non-genetic factors that interact in complex ways. Similarly, lack of response to ADs treatment is very often encountered, despite continuous improvements in treatment recommendations and alternatives to convention pharmacotherapy, including psychotherapy and deep brain stimulation as well as augmenting agents (e.g., atypical antipsychotic drugs or lithium). We still do not know what is the mechanism of such diversity of responses, therefore finding biomarkers useful in the identification of patient subpopulations-potential treatment responders $v s$. nonresponders, not to mention biomarkers useful to distinguish bipolar from MDD patients-is of utmost importance.

The idea that circulating microRNAs (miRNAs) might be a good indicator of processes underlying psychiatric illnesses is present in the scientific literature for some time (2). The first miRNA was discovered in 1993 (3) while studying the development of $C$. elegans. Since then, many families of these small oligonucleotides have been identified. miRNAs molecules, widely expressed in eukaryotes, are small (17-24 nucleotides), non-coding RNA transcripts that play important role in the post-transcriptional regulation 
of many genes. miRNA binds to the 3'UTR region of targeted mRNA and causes its degradation by recruiting RISC protein complex. Animal miRNAs exhibit partial complementarity to targeted mRNA, therefore one miRNA may regulate translation of more than one protein. Over 2,500 miRNAs have been identified in human genome alone, which may target about $30 \%$ of genes. Moreover, miRNA oligonucleotides are widely expressed in all animal tissues. These molecules are present in blood plasma/serum and in other biological fluids like: saliva, cerebrospinal fluid (CSF) or sperm as extracellular nuclease-resistant entities.

Indeed, a plethora of studies dedicated to search for potential miRNA biomarkers for psychiatric and neurodegenerative disorders are available, recently reviewed by van den Berg et al. (4), and Ferrúa et al. (5). The problem however arises when one looks at the actual samples taken for analysis-it is either serum or plasma of patients, or the whole blood, or peripheral blood mononuclear cells (PBMC), or CSF. Important in this context is recent studies published by Homorogan et al. (6). The authors compared the expression of a few miRNAs in different "blood compartments": white blood cells, total plasma, exosomes from plasma and exosome depleted plasma. Samples were obtained from patients with MDD before and after 12 weeks of escitalopram treatment. This is a small pilot study, nevertheless, the obtained results point to the significant alterations in miRNA expression in different blood compartments, which one has to keep in mind while trying to draw general conclusions from various published data. The origin of extracellular miRNAs is not fully understood. It seems that they can be actively secreted by cells and in effect play an informative role between cells and tissues, and bloodstream (7). This hypothesis is explained by the presence of miRNA packed in extracellular vesicles (EVs) (exosomes) and associated with lipoproteins [highdensity lipoprotein (HDL)] in the blood. EVs are small (30-100 nm) double-membrane vesicles produced by nearly every cell type, generated by reverse budding, and usually contain cargo appropriate for parental cells. It has been shown that they play a role in communication across the blood-brain barrier between the central nervous system (CNS) and periphery (8).

Given the above-mentioned diversity in the biological samples studied to find potential biomarker(s), how can we even try to infer what happens in the brain of MDD patients...?

Due to impressive stability of circulating miRNAs in the bloodstream and often specific tissue origin, the extracellular miRNAs serve as potential non-invasive biomarkers of many diseases (including cancers) and may indeed serve as potential biomarkers for brain pathologies, including MDD $(5,9)$. Recently, the method of extracting EVs from peripheral blood samples that are derived from neurons (NDEVs) has been established (10-14) and has attracted much attention. The use of NDEVs extracted from blood samples can overcome all problems mentioned above. Studies of cargo loaded in these NDEVsmiRNAs but also possibly proteins-will bring us closer to understanding processes undergoing in the brain.

In line with this notion lies the article by Saeedi et al. entitled "Neuron-derived extracellular vesicles enriched from plasma show altered size and miRNA cargo as a function of antidepressant drug response", published recently in the Molecular Psychiatry (15). It opens up a new important avenue in the search for reliable biomarker(s) of depression (MDD) and the response to antidepressant treatment. In our opinion, this avenue should be followed and it brings some hope that samples obtained from psychiatric patients will be more unified and the results will be easier to compare.

The authors aimed to examine changes in the peripheral miRNAs associated with MDD and the response to treatment with escitalopram. The important achievement of this study was the extraction of NDEVs from the blood of MDD patients and matching controls. MDD patients were treated with escitalopram for 8 weeks, so it was also possible to distinguish treatment-responders $v s$. non-responders as far as miRNAs content in NDEVs was concerned. The enrichment for NDEVs was done with the use of L1 cell adhesion molecule (L1CAM)-biotinylated antibody, and-for the sake of comparison and verification of the miRNAs content-with synaptosome-associated protein 25 (SNAP25)-specific biotinylated antibodies. Obtained NDEVs were thoroughly characterized, especially their size (transmission electron microscopy) and miRNAs (miRNA sequencing and further analysis, including their consensus motifs and potential targets). Appropriate comparisons were done with total plasma EVs, as well as between the studied groups, i.e., MDD patients at the beginning of the study and following 8 weeks of escitalopram administration, with the additional comparison of treatment responders $v s$. nonresponders [using the Montgomery-Asberg Depression Rating Scale (MADRS)]. Additionally, the authors decided to see whether miRNAs identified within NDEVs are also present in the brain tissue (anterior cingulate cortex of suicidal victims). To make the analysis complete they also 
used in vitro models, i.e., human neural progenitor cell line (ReNcells) and HEK 293T cell line, and EVs released by these cell lines were examined.

Some important miRNAs have been identified as altered by escitalopram treatment, and three of them, miR-21$5 \mathrm{p}, \mathrm{miR}-30 \mathrm{~d}-5 \mathrm{p}$, and $\mathrm{miR}-486-5 \mathrm{p}$ were suggested to be associated with treatment response. Generally, this work can be regarded as the pattern to follow, since NDEVs are certainly the best "window" allowing us to refer to what's happening in the brain. However, one can ask further questions-why anterior cingulate cortex was chosen for comparison, especially under conditions of treatment with escitalopram, selective serotonin reuptake inhibitor, and what are the alterations induced by other ADs...? Similarly, ReNcells VM, used in the work of Saeedi et al. (15) to confirm their findings in peripheral NDEVs are generated from the ventral mesencephalon and transduced with the $m y c$ transcription factor, but one has to remember that they need to be differentiated before achieving full neuronal phenotype, characterized mostly by their electrophysiological properties. Their proteomic phenotype has been shown to be dynamic over time of differentiation (16), however, there was no information about the presence of Slc6A4 transporter (serotonin transporter essential for a mechanism of action of escitalopram). All these issues have to be taken into account before we draw more general conclusions concerning the role of NDEV-specific miRNAs, and their potential role as reliable biomarkers.

Nevertheless, looking for potential biomarkers associated with MDD and response to pharmacological treatment via examining miRNAs content in the NDEVs is the best approach. Since peripheral blood certainly contains EVs, derived from other cells functioning in the brain in concert with neurons, including astrocytes, one can imagine that similar analyses can be also undertaken on miRNA content of the EVs specific for these cells.

\section{Acknowledgments}

Funding: This work was supported by the National Science Centre, Poland (grant No. 2016/23/B/NZ4/01086).

\section{Footnote}

Provenance and Peer Review: This article was commissioned by the editorial office, $E x R N A$. The article has undergone external peer review.
Conflicts of Interest: All authors have completed the ICMJE uniform disclosure form (available at https://dx.doi. org/10.21037/exrna-21-22). The authors have no conflicts of interest to declare.

Ethical Statement: The authors are accountable for all aspects of the work in ensuring that questions related to the accuracy or integrity of any part of the work are appropriately investigated and resolved.

Open Access Statement: This is an Open Access article distributed in accordance with the Creative Commons Attribution-NonCommercial-NoDerivs 4.0 International License (CC BY-NC-ND 4.0), which permits the noncommercial replication and distribution of the article with the strict proviso that no changes or edits are made and the original work is properly cited (including links to both the formal publication through the relevant DOI and the license). See: https://creativecommons.org/licenses/by-nc-nd/4.0/.

\section{References}

1. López-Muñoz F, Alamo C. Monoaminergic neurotransmission: the history of the discovery of antidepressants from 1950s until today. Curr Pharm Des 2009; 15:1563-86.

2. Dwivedi Y. microRNAs as Biomarker in Depression Pathogenesis. Ann Psychiatry Ment Health 2013;1:1003.

3. Lee RC, Feinbaum RL, Ambros V. The C. elegans heterochronic gene lin-4 encodes small RNAs with antisense complementarity to lin-14. Cell 1993;75:843-54.

4. van den Berg MMJ, Krauskopf J, Ramaekers JG, et al. Circulating microRNAs as potential biomarkers for psychiatric and neurodegenerative disorders. Prog Neurobiol 2020;185:101732.

5. Ferrúa CP, Giorgi R, da Rosa LC, et al. MicroRNAs expressed in depression and their associated pathways: A systematic review and a bioinformatics analysis. J Chem Neuroanat 2019;100:101650.

6. Homorogan C, Enatescu VR, Nitusca D, et al. Distribution of microRNAs associated with major depressive disorder among blood compartments. J Int Med Res 2021;49:3000605211006633.

7. Xu B, Zhang Y, Du XF, et al. Neurons secrete miR-132containing exosomes to regulate brain vascular integrity. Cell Res 2017;27:882-97.

8. Balusu S, Van Wonterghem E, De Rycke R, et al. Identification of a novel mechanism of blood-brain 
communication during peripheral inflammation via choroid plexus-derived extracellular vesicles. EMBO Mol Med 2016;8:1162-83.

9. Belzeaux R, Lin R, Turecki G. Potential Use of MicroRNA for Monitoring Therapeutic Response to Antidepressants. CNS Drugs 2017;31:253-62.

10. Cha DJ, Mengel D, Mustapic M, et al. miR-212 and miR132 Are Downregulated in Neurally Derived Plasma Exosomes of Alzheimer's Patients. Front Neurosci 2019;13:1208.

11. Fiandaca MS, Kapogiannis D, Mapstone M, et al. Identification of preclinical Alzheimer's disease by a profile of pathogenic proteins in neurally derived blood exosomes: A case-control study. Alzheimers Dement 2015;11:600-7.e1.

12. Goetzl EJ, Boxer A, Schwartz JB, et al. Altered lysosomal proteins in neural-derived plasma exosomes in preclinical

doi: 10.21037/exrna-21-22

Cite this article as: Dziedzicka-Wasylewska M, Solich J, Faron-Górecka A. MicroRNAs present in neuron-derived extracellular vesicles extracted from the blood of psychiatric patients as potential biomarkers of mood disorders. ExRNA 2021;3:11.
Alzheimer disease. Neurology 2015;85:40-7.

13. Mustapic M, Eitan E, Werner JK Jr, et al. Plasma Extracellular Vesicles Enriched for Neuronal Origin: A Potential Window into Brain Pathologic Processes. Front Neurosci 2017;11:278.

14. Sun B, Dalvi P, Abadjian L, et al. Blood neuron-derived exosomes as biomarkers of cognitive impairment in HIV. AIDS 2017;31:F9-F17.

15. Saeedi S, Nagy C, Ibrahim P, et al. Neuron-derived extracellular vesicles enriched from plasma show altered size and miRNA cargo as a function of antidepressant drug response. Mol Psychiatry 2021. doi: 10.1038/s41380-02101255-2.

16. Song Y, Subramanian K, Berberich MJ, et al. A dynamic view of the proteomic landscape during differentiation of ReNcell VM cells, an immortalized human neural progenitor line. Sci Data 2019;6:190016. 